\title{
Tiny Magnetic Robot Mechanism and Manipulation for Stent Transportation and Installation
}

\author{
Chang-Ho Yu ${ }^{1}$ and Sung Hoon Kim ${ }^{2 *}$ \\ ${ }^{I}$ Department of Convergence Technology Engineering, Chonbuk National University, 567 Baekje-daero, Jeonju, \\ Jeonbuk 54896, Republic of Korea \\ ${ }^{2}$ Department of Electronics Convergence Engineering, Wonkwang University, 460 Iksandae-ro, Iksan, \\ Jeonbuk 54538, Republic of Korea
}

(Received 6 March 2017, Received in final form 15 March 2017, Accepted 16 March 2017)

\begin{abstract}
Magnetic spiral-type microrobots, which are driven by a rotating magnetic field, have excellent locomotive abilities, whereas their medical applications are limited in the terms of function, such as the ability to drill in blood vessels. In this study, we propose a new robot with superior applications using a magnetic spiral-type machine. The proposed robot can be applied to stent transportation and installation without a catheter. In particular, the robot can be applied to the cardiovascular system, cerebrovascular disease, and nonvascular stent applications depending on the robot size. The robot consists of two independent spiral-type machines and four magnets in total. We controlled directions of thrust force of the two machines, respectively, for active locomotion with a task. We conducted a preliminary validation of the proposed robot for stent transportation and installation through experimental analyses.
\end{abstract}

Keywords : magnetic robot, stent transportation, spiral-type machine, minimally invasive treatment

\section{Introduction}

Multiscale magnetic robots and their magnetic manipulation systems are innovative approaches for the diagnosis and therapy of minimally invasive medicine [15]. The best advantage of magnetic robots is wireless control and being battery-free on the body because of magnetic field controls. In addition, the multi-scale fabrication of magnetic robots is provided up to micro/nano scales $[2,6]$. Medical magnetic robots have been applied to targeted drug delivery, drilling in blood vessels, and a robotic capsule-endoscope for diagnosis and therapy [710].

One representative application for minimally invasive medicine is a stent application. Typically, stents are divided into five classes: the coronary stent, peripheral stent, neurovascular stent, stent graft, and nonvascular stent. In particular, a cerebral aneurysm rupture has been one of the most dangerous disorders threatening people's health, and with those current medical facilities, aneurysms are

CThe Korean Magnetics Society. All rights reserved.

*Corresponding author: Tel: +82-63-850-6739

Fax:+82-63-850-6739, e-mail: kshoon@wku.ac.kr possible to be detected before a rupture. Endovascular intervention is a therapeutic minimally invasive approach to treating a cerebral aneurysm from inside the vessel by using various interventional devices (guidewire, catheter, balloon, stent, and so on). As the process of the treatment of an aneurysm also has a high risk that may cause severe damage to the patient, medical doctors are faced with a hard decision of whether to treat those aneurysms or not [11]. In addition, it is very difficult for medical doctors to know advanced techniques and get experience. To overcome these issues, a new approach is required. In this paper, we propose a new solution for stent transportation and installation using a magnetic robot based on a magnetic spiral-type mechanism without interventional devices. The performances of previous magnetic spiral-type machines have been reported [12-14]. The proposed robot consists of both left-handed and the right-handed screw mechanisms. Each spiral-type machine includes two magnets to generate propulsive forces and attractive force between two robot bodies, respectively. The robot is driven within a three axis Helmholtz coil system using magnetic torque (a uniform rotating magnetic field). Using the manipulation system, we conducted various experimental analyses and verified the capability of the active 
locomotion of the robot mechanism and stent transportation through installation in a silicone tube with air.

\section{Robot Mechanism and Principle of Manipulation}

The proposed robot consists of two independent spiraltype machines on the robot body (both the left-handed and right-handed screw mechanism, respectively). Each spiral-type machine includes two magnets to generate propulsive forces $\boldsymbol{F}_{p 1}$ and $\boldsymbol{F}_{p 2}$ from M1 and M4 with attractive force $\boldsymbol{F}_{\text {att }}$ between M2 and M3, respectively, as shown in Fig. 1(a). For stent transportation, the robot utilized directions of the thrust force of the two spiral mechanisms on the robot body. Because the installed magnets (M1 and M4) have a radial direction of a magnetic moment, the two spiral-type machines are synchronized by the applied rotating magnetic field. Under this condition, a clockwise rotating magnetic field causes both the clockwise $(\mathrm{CW})$ rotation of machine1 and the counter-clockwise $(\mathrm{CCW})$ rotation of machine2, respectively. Thus, the generated thrust forces $\left(\boldsymbol{F}_{p 1}\right.$ and $\left.\boldsymbol{F}_{p 2}\right)$ are in the opposite directions, as shown in Figs. 1(a-1) and (a-2).

The applied rotating magnetic field (CW) produces forward locomotion because the blade pitch $\mathrm{P}_{1}$ on machine 1 is shorter than $\mathrm{P}_{2}$ on machine. In addition, magnetic torque of the machine 1 is higher than that of the machine 2 at the same angle $\theta$ between the magnetization and the applied field when the two machines are synchronized by the applied field.

Therefore, the total propulsive force of the forward locomotion becomes $\boldsymbol{F}_{p}=\boldsymbol{F}_{p 1}-\boldsymbol{F}_{p 2}$. On the other hand, the applied rotating magnetic field $(\mathrm{CCW})$ results in a separation of the robot body because of each direction of the machine's propulsive force. Then, the transported stent will be installed at a target position. Under the condition, the combined force direction $\left(\boldsymbol{F}_{p 1}\right.$ and $\left.\boldsymbol{F}_{p 2}\right)$ is like repulsive force, which is higher than $\boldsymbol{F}_{\text {att }}$. The designed robot has the same mechanical properties as the spiral mechanism, whereas the magnetic moments of M1 and M4 are different to create a different torque with propulsive force for active locomotion using magnetic torque control within the rotating magnetic field. The generated magnetic torque can be expressed as follows:

$$
\boldsymbol{T}=\boldsymbol{M} \times \boldsymbol{B}_{\text {ext }},
$$

where $\boldsymbol{M}$ is the magnetic moment and $\boldsymbol{B}_{\text {ext }}$ is the applied external magnetic field density. When the robot acts forward locomotion at $\mathrm{X}$ axis and the plane of the rotating magnetic field is changed from the $\mathrm{YZ}$ plane to the $\mathrm{XYZ}$ plane, the driving rotating magnetic field density can be expressed as follows [15]:

$$
\mathbf{B}_{x, y, z}=\left(\begin{array}{c}
B_{x} \\
B_{y} \\
B_{z}
\end{array}\right)=\mu_{0} H_{0}\left(\begin{array}{c}
\cos \zeta \sin \alpha \sin \omega t-\sin \zeta \cos \omega t \\
\cos \zeta \cos \omega t+\sin \zeta \sin \alpha \sin \omega t \\
\cos \alpha \sin \omega t
\end{array}\right),
$$

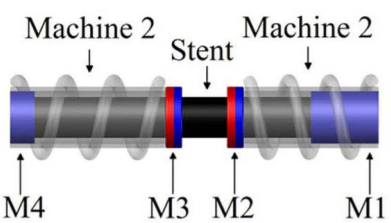

(1)

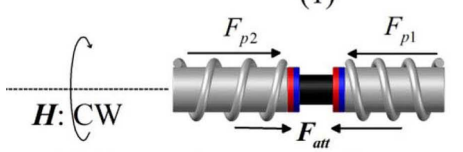

(2) Forward movement $\left(\boldsymbol{F}_{p}=\boldsymbol{F}_{p 1}-\boldsymbol{F}_{p 2}\right)$

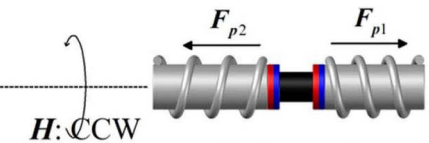

(3) Seperation

(a)

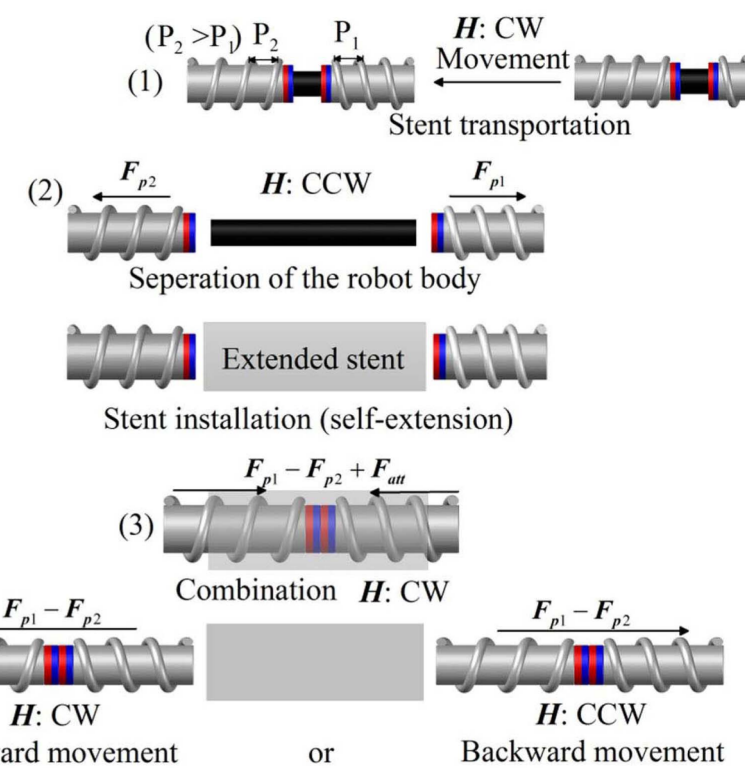

(b)

Fig. 1. (Color online) (a) Robot mechanism: (1) conceptual design and configuration of the robot body (initial condition); (2) mechanism of forward movement with stent transportation; (3) mechanism of separation of the body for stent installation; and (b) control procedure of the robot for stent transportation and installation. 
The driving magnetic torque (Eq. 1) for 3D locomotion can be rewritten as follows:

$$
\begin{aligned}
& \mathbf{T}=\mu_{0} \mathbf{m} \times \mathbf{H}_{x, y, z} \\
& \mathbf{T}=\mu_{0} m_{0} H_{0}\left\{\begin{array}{l}
(\cos \alpha \cos \theta \sin \omega t-\sin \theta(\cos \zeta \cos \omega t \\
+\sin \zeta \sin \alpha \sin \omega t)) \mathbf{i} \\
-\sin \theta(\sin \zeta \cos \omega t-\cos \zeta \sin \alpha \sin \omega t) \mathbf{j} \\
+\cos \theta(\sin \zeta \cos \omega t-\cos \zeta \sin \alpha \sin \omega t) \mathbf{k}
\end{array}\right\},
\end{aligned}
$$

where $m_{0}$ is a magnitude of the magnetic moment: $\mathbf{m}=$ $\left(0, \boldsymbol{m}_{0} \cos \theta, \boldsymbol{m}_{0} \sin \theta\right) . \mathbf{H}$ is a rotating magnetic field with strength $H_{0}$ and the angular velocity $\omega$ of the current source: $\mu_{0}$ denotes the permeability of free space. $\alpha$ and $\zeta$ represent the zenith and azimuth angles of the plane of the magnetic field. In addition, mechanical thrust force of the robot is expressed as follows:

$$
\boldsymbol{F}_{\boldsymbol{t}}=\boldsymbol{F}_{\boldsymbol{n}} \cos \theta_{\boldsymbol{n}} \cos \alpha-\boldsymbol{F}_{\boldsymbol{f}} \sin \alpha
$$

where $\boldsymbol{F}_{f}$ is the friction force opposing the movement on the thread surface: $\boldsymbol{F}_{\boldsymbol{f}}=\mu_{s} \cdot \boldsymbol{F}_{\boldsymbol{n}}, \boldsymbol{F}_{\boldsymbol{n}}$ is the reactive force $\left(\boldsymbol{F}_{\boldsymbol{n}}\right.$ $=\boldsymbol{F}_{\boldsymbol{t}} /\left(\cos \theta_{n} \cos \alpha-\mu_{s} \sin \alpha\right)$, and $\theta_{n}$ and $\alpha$ is the thread angle and the lead angle, respectively. Magnetic attractive force $\left(\boldsymbol{F}_{\text {att }}\right)$ between M2 and M3 can be analyzed by Gillbert model. In this method, $\boldsymbol{F}_{\text {att }}$ can be expressed as follows [15]:

$$
F \approx-\frac{1}{4} \pi M_{s}^{2} R^{4} \sum_{i j=0}^{1} \frac{(-1)^{i+j}}{\left(x+i t_{1}+j t_{2}\right)^{2}}\left[1-\frac{3}{2} \frac{r^{2}}{\left(x+i t_{1}+j t_{2}\right)^{2}}\right]
$$

where $M_{s}$ is the magnetization, $R$ is the magnets radius, $x$ is the distance between magnets, $t$ is the thickness of the magnet, and $\mathrm{r}$ is the lateral displacement. If $t_{1}=t_{2}=t$ and $r=0$ then $\boldsymbol{F}$ can be rewritten as follows:

$$
F \approx-\frac{1}{4} \pi M_{s}^{2} R^{4}\left[\frac{1}{x^{2}}+\frac{1}{(x+2 t)^{2}}-\frac{2}{(x+t)^{2}}\right]
$$

When $t<<x$, Eq. (5) is reached at $F \approx 3 \pi \mu_{0} M_{s}^{2} R^{4} t^{2} / 2 x^{4}$. Figure 1(b) illustrates the procedure of active locomotion and stent transportation with installation by controlling the rotating magnetic field. To reach the target point, we apply a rotating magnetic field $(\mathrm{CW})$ to the robot. Then, the direction of the forces $\boldsymbol{F}_{p 1}$ and $\boldsymbol{F}_{p 2}$ with $\boldsymbol{F}_{\text {att }}$ maintains the combined robot body during forward movement, as shown in Fig. 1(b-1), whereas the applied field $(\mathrm{CCW})$ produces an opposite direction of the $\boldsymbol{F}_{p 1}$ and $\boldsymbol{F}_{p 2}$. When the generated forces $\left(\boldsymbol{F}_{p 1}\right.$ and $\left.\boldsymbol{F}_{p 2}\right)$ are greater than $\boldsymbol{F}_{a t}$, the robot body is separated and the stent is installed through self-extension, as shown in Fig. 1(b2). The extended stent has a wider diameter than that of the robot body. Therefore, the extended stent becomes the moving path of the robot after installation. After stent

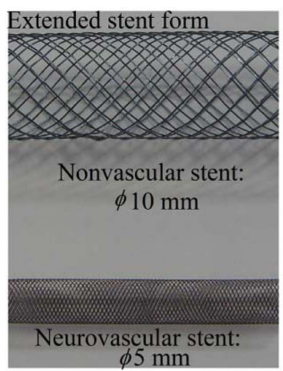

(a)

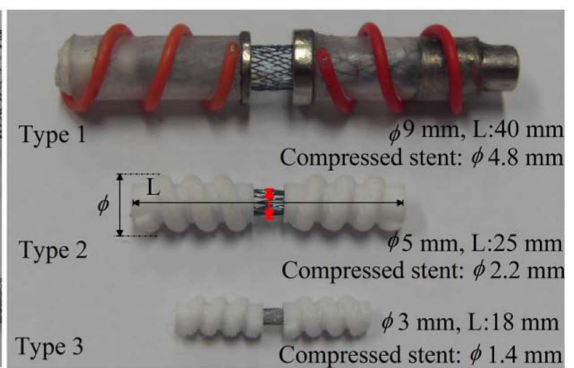

(b)
Fig. 2. (Color online) (a) Types of stents: the extended stents; (b) prototypes of the robot.

installation, the applied field (CW) results in the recombination of the two separated bodies because of the changed direction of forces $\left(\boldsymbol{F}_{p 1}\right.$ and $\left.\boldsymbol{F}_{p 2}\right)$. Furthermore, the attractive force $\left(\boldsymbol{F}_{\text {att }}\right)$ between M2 and M3 is acting and increasing to combine the two machines, when the distance between the two machines is closer for the recombination. The forward or backward movement of the recombined robot is determined by the applied rotating magnetic field $\mathrm{CW}$ or CCW, respectively, as shown in Fig. 1(b-3 and 4). The robot can be applied to vascular and nonvascular stent applications according to the robot size. Therefore, we fabricated three prototypes of the robot, as shown in Fig. 2. Figure 2(a) shows the utilized two self-extended type stents (nonvascular and vascular stents); the extended diameters of nonvascular and vascular stent are $10 \mathrm{~mm}$ and $5 \mathrm{~mm}$, respectively. Hence, the diameters of the robot are less than those of the stents. We implemented three types of robots according to the robot size, as shown in Fig. 2(b).

Type 1 and 2 included a nonvascular stent and Type 3 installed a vascular stent. In case of Type 1, the length and diameter are $40 \mathrm{~mm}$ and $9 \mathrm{~mm}$, respectively. Spiral pitch $P_{1}$ is $3.5 \mathrm{~mm}$ on the machine 1 and $\mathrm{P}_{2}$ is $4.5 \mathrm{~mm}$ on the machine 2. Table 1 shows the specification of the four magnets in Type 1 of the robot. M1 and M4 generate the rotation of the two machines by magnetic torque; the

Table 1. Specification of four magnets at prototype (Type 1).

\begin{tabular}{lcccc}
\hline \hline & M1 & M2 & M3 & M4 \\
\hline Diameter [mm] & 4.8 & $\begin{array}{c}\text { Inner: 4.5 } \\
\text { Outer: } 8\end{array}$ & $\begin{array}{c}\text { Inner: 4.5 } \\
\text { Outer: } 8\end{array}$ & 3.4 \\
Length [mm] & 10 & 1 & 2 & 7.7 \\
$\begin{array}{l}\text { Magnetization } \\
\text { (M) }\left[\mathrm{Am}^{2}\right]\end{array}$ & 0.0492 & 0.01 & 0.0056 & 0.0176 \\
$\begin{array}{l}\text { Direction of M } \\
\text { Material }\end{array}$ & Radial & Axial & Axial & Radial \\
Type & Nd-Fe-B & Nd-Fe-B & Nd-Fe-B & Nd-Fe-B \\
& Cylindrical & ring & ring & Cylindrical \\
\hline
\end{tabular}


magnetic moments of M1 and M4 are $0.0492 \mathrm{Am}^{2}$ and $0.0176 \mathrm{Am}^{2}$, respectively. Under the condition, the thrust force of machine 1 is higher than that of machine 2 for forward movement. In addition, an air gap of $6 \mathrm{~mm}$ between the two machines produces a constant attractive force to maintain separation during forward movement. In addition, the force accelerates the recombination of the separated body after stent installation.

\section{Experimental Analysis}

We conducted various performance evaluations within an electromagnetic manipulation system based on three axis Helmholtz coils, as shown in Fig. 3. In the experiments, the robot of Type 1 is performed in a silicone tube without liquid. Therefore, the robot depends on the friction between the threads on the robot body and inner wall in the silicone tube for active locomotion and separation of the robot body. If the robot is driven in liquid, the friction between the machines and stent is important factor for stent installation. For the preliminary validation, we utilized an electromagnetic manipulating system based on the three-axis Helmholtz coil, where the robot is controlled by magnetic torque. First, we investigated the thrust force of forward movement according to changes in the magnetic field strength $(4.77,6.36$, and $7.95 \mathrm{kA} / \mathrm{m})$ and frequency $(2,4,6,8$, and $10 \mathrm{~Hz})$, where the direction of the rotating magnetic field is $\mathrm{CW}$. Under these condi-

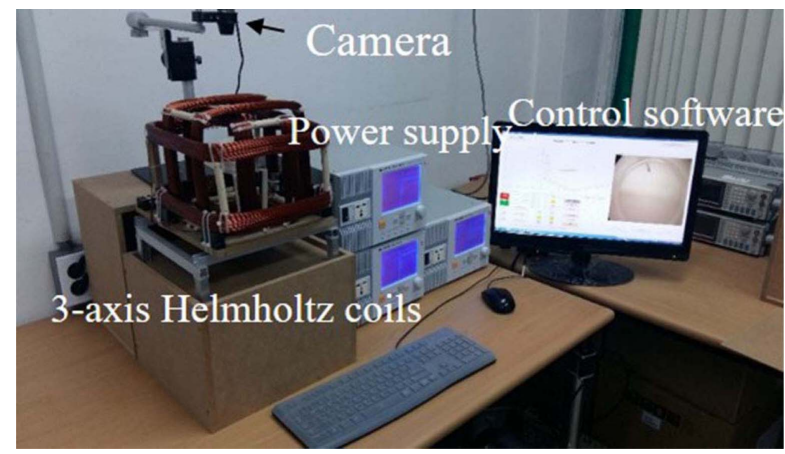

Fig. 3. (Color online) Experimental apparatus: 3 axis electromagnetic manipulation system.

tions, the robot created a maximum thrust force $\left(\boldsymbol{F}_{\text {thrust }}\right)$ of $0.097 \mathrm{~N}$ at $10 \mathrm{~Hz}$ and $7.95 \mathrm{kA} / \mathrm{m}$, and the minimum $\boldsymbol{F}_{\text {thrust }}$ is $0.0094 \mathrm{~N}$ at $2 \mathrm{~Hz}$ and $4.77 \mathrm{kA} / \mathrm{m}$, as shown in Fig. 4(a). The total thrust force $\left(\boldsymbol{F}_{\text {thrust }}\right)$ for forward locomotion becomes $\boldsymbol{F}_{\mathrm{P} 1}-\boldsymbol{F}_{\mathrm{P} 2}$. A higher frequency and higher magnetic field causes an increase in thrust force.

In the equivalent driving conditions, we observed movement velocity, as shown in Fig. 4(b). In the results, increasing ration of movement velocity depended on the driving frequency rather than variations in magnetic field strength. The applied magnetic field determines a step-out point. In the results, the robot was not fully synchronized because of sliding motion at the starting point. Thus, the movement velocities were different at each frequency

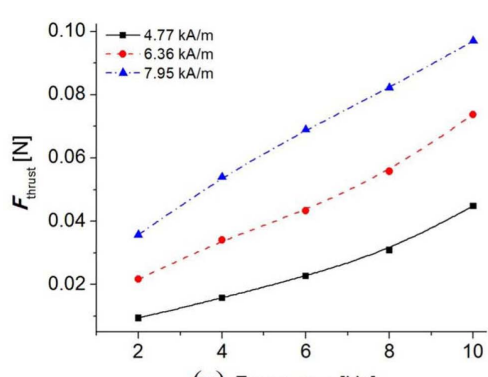

(a) Frequency $[\mathrm{Hz}]$

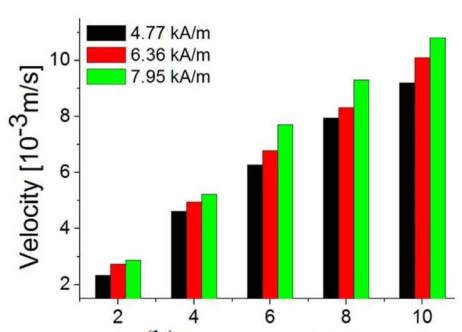

(b) Frequency $[\mathrm{Hz}]$

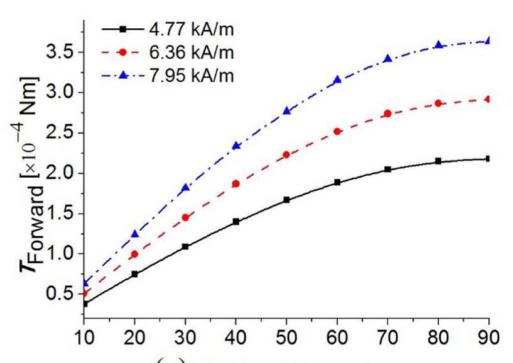

(c) Angle $\theta$ [degrees]

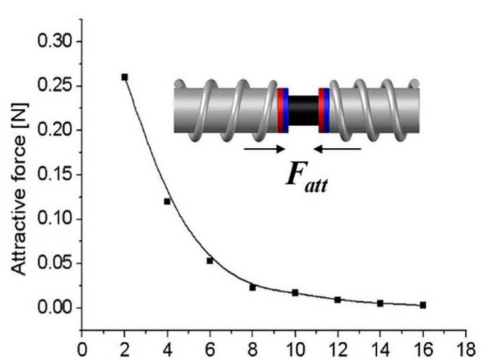

(d) Distance $[\mathrm{mml}$

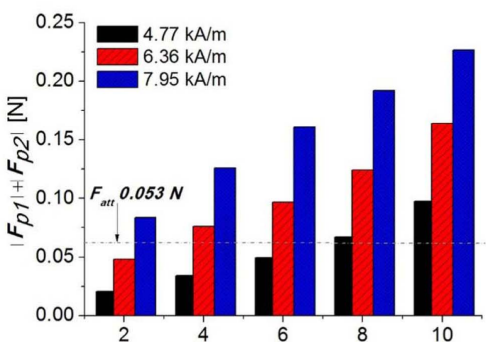

(e) Frequency [Hz]

Fig. 4. (Color online) (a) Observation of total thrust force for forward movement; (b) movement velocity of forward movement; (c) variations in the driving magnetic torque according to changes an angle $\theta$ between the magnetic field and the direction of magnetic moment; (d) An attractive force between M2 and M3 according to changes in air gap; (e) force relationship between thrust forces and attractive force for separation of the robot body. 
according to changes in the magnetic field.

When we changed the driving frequency, a maximum difference between $2 \mathrm{~Hz}$ and $10 \mathrm{~Hz}$ was 3.95 times at $4.77 \mathrm{kA} / \mathrm{m}$, whereas a maximum difference in the velocity between 4.77 and $7.95 \mathrm{kA} / \mathrm{m}$ was 1.17 times the fixed frequency of $10 \mathrm{~Hz}$. Under the observation, the driving frequency up to $10 \mathrm{~Hz}$ showed an increase in the velocity, while the exceeded $10 \mathrm{~Hz}$ resulted in unsynchronized rotation and a slightly declined velocity because of a decrease in magnetic torque. If the robot produces sufficient torque, the robot will show synchronized rotation with increasing velocity at exceeding $10 \mathrm{~Hz}$. For active locomotion, the robot has operational ranges, when magnetic field is between $4.77 \mathrm{kA} / \mathrm{m}$ and $7.95 \mathrm{kA} / \mathrm{m}$ and frequency is up to $10 \mathrm{~Hz}$. We estimated the magnetic torque using Eq. (1) according to changes in an angle $\theta$ up to $90^{\circ}$ at the fixed magnetic moment. Minimum driving magnetic torque was $0.38 \times 10^{-4} \mathrm{Nm}$ at $\theta$ of $10^{\circ}$ and 4.77 $\mathrm{kA} / \mathrm{m}$. Maximum magnetic torque was $3.64 \times 10^{-4} \mathrm{Nm}$ at $\theta$ of $90^{\circ}$ with $7.95 \mathrm{kA} / \mathrm{m}$, as shown in Fig. 4(c). The results showed the establishment of driving torque range for forward movement.

In the robot mechanism, one of the remarkable mechanisms is the attractive force $\left(\boldsymbol{F}_{\text {att }}\right)$ between M2 and M3. $\boldsymbol{F}_{\text {att }}$ performs two functions to prevent separation of the two bodies during forward movement and to accelerate recombination of the separated body after stent installation. In the case of Type 1, the initial distance between M2 and $\mathrm{M} 3$ is $6 \mathrm{~mm}$ and its attractive force is $0.053 \mathrm{~N}$. Figure 4 (d) shows variations in attractive force according to changes in the air gap between M2 and M3 (up to 16 $\mathrm{mm})$. A $\boldsymbol{F}_{\text {att }}$ of $0.053 \mathrm{~N}$ becomes the threshold for separation of the robot body. If thrust forces of the two machines are less than $0.053 \mathrm{~N}$ within the rotating magnetic field $(\mathrm{CCW})$, the robot body cannot be separated.
Figure 4(e) shows the relationship between $\boldsymbol{F}_{\text {att }}$ and the thrust forces of the two machines within a rotating magnetic field $(\mathrm{CCW})$ according to changes in the driving frequency. The increase in the magnetic field results in the increased driving frequency because the increased magnetic field causes an increase in magnetic torque up to step-out point. Therefore, a higher driving magnetic field provides a wider driving range of the driving frequency for separation of the body. To separate the robot body, sum of the two thrust force must exceed $0.053 \mathrm{~N}$ because $0.053 \mathrm{~N}$ is an attractive force between M2 and M3. The driving magnetic field of $7.95 \mathrm{kA} / \mathrm{m}$ allows the separation of the body at the driving frequency of $2 \mathrm{~Hz}$, whereas 4.77 and $6.36 \mathrm{kA} / \mathrm{m}$ cannot separate the robot body at $2 \mathrm{~Hz}$. When the driving frequency was reached at $8 \mathrm{~Hz}$, the robot body can be separated at a low driving magnetic field of $4.77 \mathrm{kA} / \mathrm{m}$.

We conducted a preliminary validation of stent transportation with installation, as shown in Fig. 5. In the experiment, the robot was driven by a magnetic field of $7.95 \mathrm{kA} / \mathrm{m}$ and a frequency of $2 \mathrm{~Hz}$. Figures 5 (a) and (b) shows the forward movement by the $\mathrm{CW}$ the rotating magnetic field. When the robot reached the desired position, the rotating magnetic field is changed from $\mathrm{CW}$ to $\mathrm{CCW}$ for separation of the robot (Fig. 5(c)). Then, the stent is extended by self-extension. Next, the applied magnetic field is changed from $\mathrm{CCW}$ to $\mathrm{CW}$ for the recombination of the robot, and $\boldsymbol{F}_{\text {att }}$ accelerates the recombination. Because of the increased $\boldsymbol{F}_{\text {att }}$, the recombined time was 1 s (Fig. 5(d-e)). Finally, the CCW rotating magnetic field caused backward movement (Fig. 5(f)).

\section{Discussion and Conclusion}

In this study, we proposed a new magnetic robot

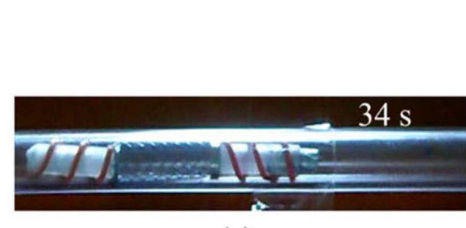

(c)

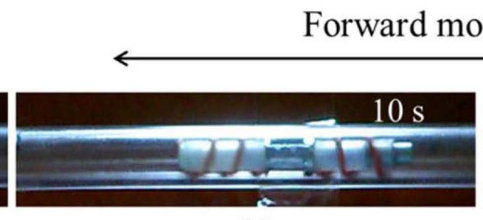

(b)

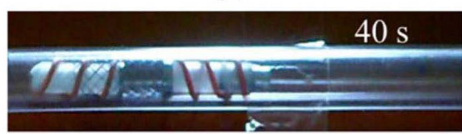

(d)

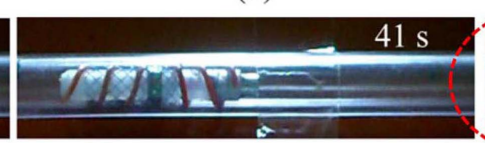

(e)

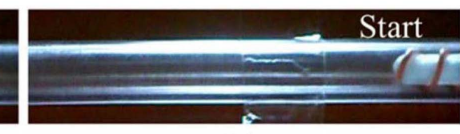

(a)

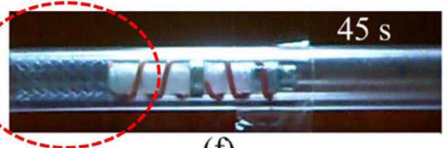

(f)

Backward movement

Fig. 5. (Color online) Robot manipulation: (a) to (c) are forward movements by a CW magnetic field of $7.95 \mathrm{kA} / \mathrm{m}$ and a driving frequency of $2 \mathrm{~Hz}$ in a silicone tube; (c) separation of the robot body and the extended stent within the CCW magnetic field for stent installation; (d) and (e) are recombination of the robot body by the CCW magnetic field; (f) return to the starting point by the CCW magnetic field. 
mechanism for stent transportation and installation using magnetic wireless manipulation for minimally invasive treatment. Through an external magnetic field, the robot was controlled by magnetic torque, which determines the direction of thrust forces of machines 1 and 2, respectively. In addition, the two magnets (M2 and M3) are creating an attractive force for the recombination of the separated body. To verify the proposed mechanisms and a facility for stent transportation with installation, we performed various experimental analyses. Although we could not conduct an in-vivo test, we were able to verify the preliminary validity of the robot mechanism. Vascular robots have potential risk if not precisely controlled. In particular, if the robot scratches cerebrovascular inner wall, it is fatal to life because cerebrovascular inner wall is very thin. However, if the robot apply to cardiovascular system or nonvascular system, the scratch is not fatal to life. When we consider the robot size, the application of the robot is suitable for use in cardiovascular system or nonvascular system.

Our ongoing work is focused on developing the enhanced mechanism for actual clinical application and precision control. In addition, we will conduct the experiments in various environments (fluid flow and various viscosities) to investigate mobility. Through future works, we will develop an advanced robot based on the mechanism. The robot will be applied to multiple functions, such as diagnosis and therapy through a combination of drilling or a capsule-type endoscope or drug delivery.

\section{Acknowledgments}

This research was supported by Wonkwang University in 2017.

\section{References}

[1] J. J. Abbott, K. E. Peyer, M. C. Lagomarsino, L. Zhang, L. X. Dong, I. K. Kaliakatsos, and B. J. Nelson, Int. J. Robot. Res. 28, 1434 (2009).

[2] A. Ghosh and P. Fischer, Nano Lett. 9, 2243 (2009).

[3] B. J. Nelson, I. K. Kaliakatsos, and J. J. Abbott, Annual Review of Biomedical Engineering 12, 55 (2010).

[4] S. H. Kim and K. Ishiyama, IEEE/ASME Trans. Mechatronics 19, 1651 (2014).

[5] C. H. Yu and S. H. Kim, J. Magn. 21, 616 (2016).

[6] L. Zhang, J. Abbott, L. Dong, K. D. Bell, and B. J. Nelson, Appl. Phys. Lett. 94, 064017 (2009).

[7] S. M. Jeong and G. H. Jang, IEEE Trans. Magn. 48, 4062 (2012).

[8] S. Pane, O. Ergeneman, K. M. Sivaraman, T. Luhmann, H. Hall, and B. J. Nelson, Proc. IEEE Int' Conf. Nano/ Molecular Medicine and Engineering (2010) pp. 148152.

[9] S. Yim and M. Sitti, IEEE Trans. Robotics 28, 183 (2012).

[10] M. Sendoh, K. Ishiyama, and K. I. Arai, IEEE Trans. Magn. 39, 3232 (2003).

[11] Y. H. Kim, X. Xu, and J. S. Lee, Annals of Biomedical Engineering 38, 2274 (2010).

[12] K. Ishiyama, M. Sendoh, A. Yamazaki, and K. I Arai, Sensors and Actuators A 91, 141 (2001).

[13] S. H. Kim, K. S. Shin, S. Hashi, and K. Ishiyama, IEEE Trans. Magn. 49, 3488 (2013).

[14] S. H. Kim, J. W. Shin, and K. Ishiyama, IEEE Trans. Magn. 50, 9100404-1-4 (2014).

[15] C. H. Yu and S. H. Kim, J. Magn. 21, 616 (2016).

[16] D. Vokoun, M. Beleggia, L. Heller, and P. Sittner, J. Magn. Magn. Mater. 321, 3758 (2009). 